\title{
FORZE PONDEROMOTRICI ESERCTTATE DA UN CAMPO MAGNETICO OMOGENEO SU UNA CORRENTE CONTINUA RETTILINEA INDEFINITA
} DI BRUTO CALDONAZZO.

\section{Forze ponderomotrici.}

Le azioni che un campo elettromagnetico esercita sulle masse elettriche e magnetiche situate in un corpo si manifestano-come azioni meccaniche agenti sulla massa del corpo stesso ; e per questo le forze corrispondenti a tali azioni prendono il nome di «forze ponderomotrici ».

Maxwell ", si propose la determinazione di tali forze assimilandole a tensioni interne tendenti a deformare il mezzo; perciò spesso vengono indicate col nome di tensioni maxvelliane.

Hertz ${ }^{3}$ ) invece ammettendo per tali forze il principio della azione eguale ed opposto alla reazione ed il principio della conservazione dell' energia determinò le forze ponderomotrici partendo dalle sue equazioni fondamentali della elettrodinamica per un corpo in movimento.

Senza entrare in discussione sopra la maggiore o minore attendibilità del risultato ottenuto dal primo o dal secondo dei due grandi fisici matematici mi sono proposto la determinazione tanto secondo Maxwell che secondo Hertz delle forze ponderomotrici- che un campo magnetico omogeneo esercita sopra una corrente continua in un conduttore cilindrico indefinito. Dapprima, date le espressioni di tali forze pel caso generale, accenno che per altra via e senza avvertirlo Ein-

1) Maxwell. « Treatise » II, pag. 254 (1873).

2) Hertz. Ann. der Phys. 41, pag. 389 (1890), oppure Untersuchungen, pag. 234 e 275 (1895). 
sein e Laub ') diedero per queste forze espressioni che coincidono con quelle di Maxwell.

Dalle espressioni in forma vettoriale delle forze ponderomotrici agenti per unità di volume deduco le espressioni delle equivalenti pressioni fittizie in superficie. Determinato poi il campo magnetico risultante all' esterno ed all' interno del conduttore applico direttamente le formule di Maxwell e di Hertz. Ottengo quindi la forza risultante agente su ogni porzione di cilindro conduttore di lunghezza 1 (e che indicherò in seguito col nome di sezione unitaria) integrando su questa porzione la forza di volume agente all' interno del conduttore per unità di volume e aggiungendo a questo primo contributo il contributo portato dalla discontinuità delle pressioni fittizie in superficie. Accenno poi come per altra via si poteva calcolare questa risultante; infine confronto i due diversi valori ottenuti.

\section{Forze ponderomotriei esercitate da un eampo magnetico su una corrente.}

Secondo Maxwell la forza esercitata su l'unità di volume di un corpo magnetizzato con una intensità di magnetizzazione $\mathbf{M}$, da un campo magnetico di forza $\mathbf{H}$ è secondo l'asse delle $x$

$$
\mathbf{M}_{x} \frac{\partial \mathbf{H}_{x}}{\partial x}+\mathbf{M}_{y} \frac{\partial \mathbf{H}_{y}}{\partial y}+\mathbf{M}_{z} \frac{\partial \mathbf{H}_{z}}{\partial z}
$$

ossia, essendo

$$
\mathbf{M}=\frac{\mu-1}{4 \pi} \mathbf{H},
$$

questa forza ̀̀ rappresentata dal vettore

$$
\frac{\mu-1}{8 \pi} \operatorname{grad} \mathbf{H}^{2},
$$

essendo $\mu$ la permeabilità magnetica del mezzo.

1) Einstein und Laub. Ann. der Phys. 26, pag. 544 (1908). 
Se poi il corpo magnetizzato è percorso da una corrente elettrica di densità $\mathbf{I}$ si deve aggiungere alla forza (1) la forza

$$
\frac{1}{c}[\mathbf{I B}]
$$

in cui

$$
\mathbf{B}=\mu \mathbf{H}
$$

e $c$ indica la velocità di propagazione della luce nell'etere.

Quindi complessivamente la forza agente sull'unità di volume $\dot{\theta}$

$$
\mathbf{F}=\frac{\mu-1}{8 \pi} \operatorname{grad} \mathbf{H}^{2}+\frac{1}{c}[\mathbf{i} \mathbf{B}] .
$$

Supponendo che questa forza determini nel mezzo uno stato di tensione otteniamo come componenti speciali della forza agente sull'unità di superficie perpendicolare all' asse delle $x$

$$
\begin{aligned}
& \mathrm{X}_{x}=\frac{1}{4 \pi}\left(\mathbf{H}_{x} \mathbf{B}_{x}-\frac{1}{2} \mathbf{H}^{z}\right) \\
& \mathrm{X}_{y}=\frac{1}{4 \pi} \mathbf{H}_{x} \mathbf{B}_{y}, \\
& \mathrm{X}_{z}=\frac{1}{4 \pi} \mathbf{H}_{x} \mathbf{B}_{z}
\end{aligned}
$$

essendo $\mathrm{X}_{y}=\mathrm{Y}_{z}$ ecc.

Secondo Einstein e Laub invece la forza fornita dal campo magnetico in luogo della (1) ̀̀

$$
\text { (II, grad) } \mathbf{H}
$$

e quella che si deve aggiungere se il corpo è percorso da corrente di densità i, secondo la legge di Biot e Savart è

$$
\frac{1}{c}[\mathbf{i} \mathbf{H}] \text {. }
$$

In luogo della (3) abbiamo allora

$$
\mathbf{F}=(\mathbf{M}, \operatorname{grad}) \mathbf{H}+\frac{1}{c}[\mathbf{i} \mathbf{H}] .
$$


Per dimostrare che la (3a) coincide con la (3) osserviamo chè è

$$
\text { (II, grad) } \mathbf{H}_{x}=\frac{\mu-1}{4 \pi}\left(\mathbf{H}_{2} \frac{\partial \mathbf{H}_{x}}{\partial x}+\mathbf{H}_{y} \frac{\partial \mathbf{H}_{x}}{\partial y}+\mathbf{H}_{\mathbf{z}} \frac{\partial \mathbf{H}_{x}}{\partial \boldsymbol{z}}\right)
$$

$$
=\frac{\mu-1}{4 \pi}\left(\frac{1}{2} \frac{\partial \mathbb{W}^{2}}{\partial x}-[\mathbf{H} \operatorname{rot} \mathbf{H}]_{x}\right)
$$

e quindi

$$
(\mathbf{M}, \operatorname{grad}) \mathbf{H}=\frac{\mu-1}{8 \pi}\left(\operatorname{grad} \mathbf{H}^{2}+2[\operatorname{rot} \mathbf{H}, \mathbf{H}]\right) .
$$

Essendo poi ${ }^{1}$ )

$$
\operatorname{rot} H=-\frac{4 \pi}{c} \mathbf{i}
$$

si ha subito

$$
(\mathbf{I}, \operatorname{grad}) \mathbf{H}=\frac{\mu-1}{8 \pi} \operatorname{grad} \mathbf{H}^{2}+\frac{1}{c}[\mathbf{i} \mathbf{B}]-\frac{1}{c}[\mathbf{i} \mathbf{H}],
$$

la quale ci mostra subito l'eguaglianza delle (3) alla (3a).

Secondo Hertz la forza esercitata da un campo magnetico su una corrente per unità di volume ha per componenti speciali

(4a)

$$
\mathrm{X}_{x}=\frac{1}{4 \pi}\left\{\mathbf{H}_{x} \mathbf{B}_{c}-\frac{1}{2}(\mathbf{H ~ B})\right\},
$$

$$
\mathrm{X}_{y}=\mathrm{Y}_{x}=\frac{1}{4 \pi} \mathbf{H}_{x} \mathbf{B}_{y}, \text { ecc. }
$$

Le componenti di questa forza secondo l'asse delle $x$

$$
\mathbf{F}_{x}=\frac{\partial \mathbf{X}_{x}}{\partial x}+\frac{\partial \mathbf{X}_{y}}{\partial y}+\frac{\partial \mathbf{X}_{z}}{\partial z}
$$

assume per le (4a) la forma

1) V. ad es. Abraham. " Theorie der Elektrizität , I, pag. 227 (1907). 


$$
\begin{gathered}
\mathbf{F}_{x}^{\prime}=\frac{1}{8 \pi}\left\{2 \frac{\partial}{\partial x}\left(\mathbf{H}_{x} \mathbf{B}_{x}\right)+2 \frac{\partial}{\partial y}\left(\mathbf{H}_{x} \mathbf{B}_{z}\right)+2 \frac{\partial}{\partial z}\left(\mathbf{H}_{x} \mathbf{B}_{z}\right)-\frac{\partial}{\partial x}(\mathbf{H ~ B})\right\} \\
=\frac{1}{8 \pi}\left\{2(\mathbf{B g r a d}) \mathbf{H}_{x}+2 \mathbf{H}_{x} \operatorname{div} \mathbf{B}-\frac{\partial}{\partial x}(\mathbf{H ~ B})\right\}
\end{gathered}
$$

e quindi la forza $\mathbf{F}^{\prime}$ è il vettore

$$
\mathbf{F}^{\prime}=\frac{1}{8 \pi}\{2(\mathbf{B} \operatorname{grad}) \mathbf{H}-\operatorname{grad}(\mathbf{H} \mathbf{B})\},
$$

essendo

$$
\operatorname{div} \mathbf{B}=0 .
$$

Inoltre dalla (5) ricaviamo facilmente moltiplicando per $\frac{8 \pi \mu}{\mu-1}$

$$
2(\mathbf{B}, \operatorname{grad}) \mathbf{H}=\mu \operatorname{grad} \mathbf{H}^{2}+\frac{8 \pi}{\mathrm{c}}[\mathbf{i} \mathbf{B}],
$$

ed essendo

$$
\operatorname{grad}(\mathbf{H} \mathbf{B})=\mu \operatorname{grad} \mathbf{H}^{2}+\mathbf{H}^{2} \operatorname{grad} \mu
$$

possiamo scrivere la (6)

$$
\mathbf{F}^{\prime}=\frac{1}{\mathrm{c}}[\mathbf{i} \mathbf{B}]-\frac{1}{8 \pi} \mathbf{H}^{2} \operatorname{grad} \mu .
$$

Vediamo quindi le forze $\mathbf{F}$ ed $\mathbf{F}^{\prime}$ date rispettivamente dalle $(3)$ e $(6 a)$ non coincidono e la loro differenza $\dot{\theta}$

$$
\mathbf{F}-\mathbf{F}^{\prime}=\frac{1}{2} \operatorname{grad}(\mathbf{M} \mathbf{H}) \text {. }
$$

Confrontando poi le (4) con le (4a) si vede che nelle espressioni delle $\mathrm{X}_{x}, \mathrm{Y}_{y}, \mathrm{Z}_{\tilde{z}}$ date dalle seconde in luogo di $\mathbf{H}^{2}$ compare (H B).

Determiniamo ora le forze superficiali corrispondenti rispettivamente a $\mathbf{F}$ e $\mathbf{F}$.

Consideriamo la forza $\mathbf{F}$ agente in un campo $v$ limitato dalla superficie $f$. La risultante della forza agente in questo 
campo ha per componente secondo l'asse delle $x$, tenendo conto delle (4)

$$
\begin{aligned}
\int \mathbf{F}_{x} d v=\frac{1}{4 \pi} \int d v\left\{\frac { \partial } { \partial x } \left(\mathbf{H}_{x} \mathbf{B}_{x}\right.\right. & \left.-\frac{1}{2} \mathrm{H}^{2}\right)+ \\
& \left.+\frac{\partial}{\partial y}\left(\mathbf{H}_{x} \mathbf{B}_{y}\right)+\frac{\partial}{\partial z}\left(\mathbf{H}_{x} \mathbf{B}_{z}\right)\right\} .
\end{aligned}
$$

La funzione che compare nel secondo integrale si può sempre considerare come divergenza del vettore di componenti $\mathbf{X}_{x}, \mathbf{X}_{y}, \mathbf{X}_{z} ;$ per cui indicando con $\mathbf{n}$ un vettore unitario normale alla superficie $f$ diretto all' esterno del volume $v$, pel lemma di Gauss possiamo scrivere

$$
\begin{gathered}
\int \mathbf{F}_{x} d v=\frac{1}{4 \pi} \int d f\left\{\left(\mathbf{H}_{x} \mathbf{B}_{x}-\frac{1}{2} \mathbf{H}^{2}\right) \cos n x+\mathbf{H}_{x} \mathbf{B}_{y} \cos n y+\mathbf{H}_{x} \mathbf{B}_{z} \cos n z\right\} \\
=\frac{1}{4 \pi} \int d f\left\{\mathbf{H}_{x}(\mathbf{B} \mathbf{n})-\frac{1}{2} \mathbf{H}^{2} \cos n x\right\}
\end{gathered}
$$

Alla forza $\mathbf{F}$ quindi agente sull'unità di volume possiamo sostituire una forza superficiale agente per unità di superficie

$$
\mathbf{T}=\frac{1}{8 \pi}\left\{2 \mathbf{H}(\mathbf{B ~ n})-\mathbf{H}^{2} \mathbf{n}\right\}
$$

Se consideriamo invece l'espressione della forza agente sull'unità di volume data da Hertz, con processo analogo otteniamo

$$
\begin{aligned}
\int \mathbf{F}_{x}^{\prime} d v & =\frac{1}{8 \pi} \int d v\left\{2 \frac{\partial}{\partial x}\left(\left(\mathbf{H}_{x} \mathbf{B}_{x}\right)-\frac{1}{2}(\mathbf{H ~ B})\right)+\right. \\
+ & \left.2 \frac{\partial}{\partial y} \mathbf{H}_{x} \mathbf{B}_{y}+2 \frac{\partial}{\partial z} \mathbf{H}_{x} \mathbf{B}_{z}\right\}= \\
= & \frac{1}{8 \pi} \int d f\left\{2 \mathbf{H}_{x}(\mathbf{B} \mathbf{n})-(\mathbf{B} \mathbf{H}) \cos n x\{.\right.
\end{aligned}
$$


La forza superficiale unitaria $\mathrm{e}$ in tal caso

$$
\mathbf{T}^{\prime}=\frac{1}{8 \pi}\{2 \mathbf{H}(\mathbf{B} \mathbf{n})-(\mathbf{B} \mathbf{H}) \mathbf{n}\}
$$

oppure sotto altra forma vettoriale

$$
\mathbf{T}^{\prime}=\frac{1}{8 \pi}\{\mathbf{H}(\mathbf{B} \mathbf{n})+[\mathbf{B}[\mathbf{H} \mathbf{n}]]\} .
$$

\section{Posizione del problema.}

In un campo magnetico omogeneo $\mathbf{H}_{o}=\mathbf{H}_{o z}$ si trova un conduttore cilindrico indefinito di raggio $R$, coll'asse coincidente coll'asse delle $y$. Facciamo passare pel cilindro una corrente continua di densità $\mathbf{i}=\mathbf{i}_{y}$. Determinare la forza ponderomotrice che il campo magnetico risultante esercita sul cilindro per ogni porzione di lunghezza 1 , nell'ipotesi che il mezzo esterno al cilindro e il mezzo costituente il cilindro siano isotropi e le loro permeabilità magnetiche rispettivamente $\mu_{1}$ e $\mu_{2}$ siano costanti.

\section{Determinazione del campo magnetico risultante.}

Il campo magnetico risultante è costituito dal campo primitivo $\mathbf{H}_{o}$ a cui si sovrappongono il campo indotto dalla discontinuità del mezzo sulla superficie del conduttore e il campo determinato dalla corrente. Indicando con $\varphi_{1}$ e $\varphi_{2}$ i potenziali del campo indotto rispettivamente fuori e dentro il conduttore dalla detta discontinuità, essi devono determinarsi in modo che la induzione magnetica normale e la forza magnetica tangenziale alla superficie del cilindro siano continue su questa superficie.

Queste condizioni analiticamente sono rappresentate dalle equazioni

$\mathrm{I}\left\{\begin{array}{cr}\varphi_{1}=\varphi_{3}, & \text { per } \rho=\mathrm{R} \\ \mu_{2} \frac{\partial \varphi_{2}}{\partial n}-\mu_{1} \frac{\partial \varphi_{1}}{\partial n}=\left(\mu_{3}-\mu_{1}\right) \mathrm{H}_{0} \frac{\partial z}{\partial n}, \quad \eta \quad " \quad\end{array}\right.$ 
essendo $p=\sqrt{x^{2}+y^{2}}$, ed $n$ la normale alla superficie diretta all' esterno del cilindro. A queste poi si aggiungono le condizioni indefinite

II

$$
\left\{\begin{array}{l}
\Delta_{2} \varphi_{1}=0, \quad \rho \geq R \\
\Delta_{2} \varphi_{2}=0, \quad \rho \leq R
\end{array}\right.
$$

Per ragioni di simmetria le $\varphi$ sono indipendenti da $y$; possiamo ridurre il problema quindi sul piano $x z$. Riferendo i punti di questo piano a un sistema polare di coordinate $\rho, \vartheta$. le nostre condizioni ai limiti diventano

$$
\text { (Ia) }\left\{\begin{array}{cc}
\varphi_{1}(\rho, \vartheta)=\varphi_{2}(\rho, \vartheta) & \rho=R \\
\mu_{2} \frac{\partial \varphi_{2}}{\varphi \rho}-\mu_{1} \frac{\partial \varphi_{1}}{\partial \rho}=\left(\mu_{2}-\mu_{1}\right) \mathrm{H}_{0} \operatorname{sen} \vartheta, \quad \eta \quad
\end{array}\right.
$$

mentre per le condizioni indefinite tanto $\varphi_{1}$ che $\varphi_{3}$ devono essere soluzioni della

$$
\frac{\partial^{2} \varphi}{\partial \rho^{2}}+\frac{1}{\rho} \frac{\partial \varphi}{\partial \rho}+\frac{1}{\rho^{2}} \frac{\partial^{2} \varphi}{\partial \vartheta^{2}}=0
$$

L'integrale generale di questa

$$
\varphi=\sum_{\nu=0}^{\infty}\left(A_{\nu}, \rho^{\nu}+B_{\nu}, \rho^{-\nu}\right)\left(C_{\nu} \cos \nu \vartheta+D_{\nu} \text { sen } \vee \vartheta\right)
$$

ci permette di dare a $\varphi_{1}$ e $\varphi_{2}$ le seguenti espressioni

$$
\begin{array}{ll}
\varphi_{1}=\mathrm{KH}_{o} \mathrm{R}^{2} \frac{\operatorname{sen} \vartheta}{\rho}, & \rho \geq \mathrm{R} \\
\varphi_{2}=\mathrm{KH}_{o} \rho \operatorname{sen} \vartheta, & \rho \leq \mathrm{R}
\end{array}
$$

soddisfacendo cosi alle condizioni che $\varphi_{1}$ si annulli per $\rho=\infty$ e che $\varphi_{2}$ resti finita per $\rho=0$, in cui $\dot{e}$

$$
\mathrm{K}=\frac{\mu_{2}-\mu_{1}}{\mu_{2}+\mu_{1}}
$$

soddisfacendo così alla (Ia). 
Il campo poi determinato dalla corrente non dipende da un potenziale scalare ma bensì da un potenziale vettore ${ }^{1}$ )

$$
\mathbf{A}=\frac{\mu \mathbf{i}}{c} \int \frac{d v}{r}
$$

in modo che la corrispondente induzione magnetica $\grave{e}$

$$
\mathbf{B}=\operatorname{rot} \mathbf{A} .
$$

$\mathrm{Nel}$ nostro caso $\mathbf{A}$ deve essere scelto in modo che siano soddisfatte ancora le condizioni espresse dalle (I) e (II). Introducendo ora l' intensitz di corrente $\mathbf{J}=\mathbf{J}_{y}=\pi \mathrm{R}^{2} \mathbf{i}$, i potenziali vettori pel mezzo esterno ed interno al cilindro sono rispettivamente ${ }^{2}$ )

$$
\begin{array}{ll}
\mathbf{A}_{1}=\mathbf{A}_{1 y}=-2 \mu_{1} \frac{\mathbf{J}}{c} \log \rho, & \rho \geq \mathbf{R} \\
\mathbf{A}_{2}=\mathbf{A}_{2 y}=-\mu_{z} \frac{\mathbf{J}}{c} \frac{\rho^{2}}{\mathrm{R}^{2}}, & \rho \leq \mathrm{R} .
\end{array}
$$

Abbiamo ora tutti gli elementi necessari per esprimere in base alla $\mathbf{H}=\mathbf{H}_{o}-\operatorname{grad} \varphi+\frac{1}{\mu} \operatorname{rot} \mathbf{A}$ il campo magnetico risultante internamente ed esternamente al cilindro.

Abbiamo cioè riferendoci con l'indice 1 e 2 rispettivamente al campo esterno ed interno, in coordinate cartesiane:

$$
\begin{aligned}
& \mathbf{H}_{1 x}=\mathrm{K} \mathbf{H}_{o} \frac{\mathrm{R}^{2}}{\rho^{2}} \operatorname{sen} 2 \vartheta+2 \frac{\mathrm{J}}{c} \frac{\operatorname{sen} \vartheta}{\rho} \\
& \mathbf{H}_{1 y}=0, \\
& \mathbf{H}_{1 z}=-\mathrm{KH}_{o} \frac{\mathrm{R}^{2}}{\rho^{2}} \cos 2 \vartheta-\frac{2 \mathbf{J}}{c} \frac{\cos \vartheta}{\rho}
\end{aligned}
$$

4) Abraham. L. c., pag. 227.

$\Rightarrow$ Debye. «Encykl. der Math. Wiss. V, 1T, num. 20. 
$(9 a)$

$$
\begin{aligned}
& \mathbf{H}_{2 x}=\frac{2 \mathbf{J}}{c} \frac{\rho}{\mathbf{R}^{2}} \operatorname{sen} \vartheta \\
& \mathbf{H}_{2 y}=0 \\
& \mathbf{H}_{2 z}=-(\mathrm{K}-1) \mathbf{H}_{o}-\frac{2 \mathbf{J}}{c} \frac{\rho}{\mathrm{R}^{2}} \cos \vartheta
\end{aligned}
$$

e in coordinate cilindriche:

$$
\begin{aligned}
& (10) \quad\left\{\begin{array}{l}
\mathbf{H}_{1 \rho}=\mathbf{H}_{o}\left(\mathrm{~K} \frac{\mathrm{R}^{2}}{\rho^{2}}+1\right) \operatorname{sen} \vartheta \\
\mathbf{H}_{1 y}=0, \\
\mathbf{H}_{1 \vartheta}=-\mathbf{H}_{o}\left(\mathrm{~K} \frac{\mathrm{R}^{2}}{\rho^{2}}-1\right) \cos \vartheta-\frac{2 \mathbf{J}}{c} \frac{1}{\rho} ;
\end{array}\right. \\
& (10 \mathrm{a}) \quad\left\{\begin{array}{l}
\mathbf{H}_{2 \rho}=-\mathbf{H}_{o}(\mathrm{~K}-1) \operatorname{sen} \vartheta \\
\mathbf{H}_{2 y}=0, \\
\mathbf{H}_{2 \xi}=-\mathbf{H}_{o}(\mathrm{~K}-1) \cos \vartheta-\frac{2 \mathbf{J}}{c} \frac{\rho}{\mathrm{R}^{2}} .
\end{array}\right.
\end{aligned}
$$

Sulla superficie è facile verificare ora che sono soddisfatte le condizioni ai limiti; infatti si ba per $p=R$

$$
\begin{aligned}
& \mathbf{B}_{1 \vartheta}=\mathbf{B}_{2 \rho}=\frac{2 \mu_{1} \mu_{2}}{\mu_{1}+\mu_{2}} \mathbf{H}_{o} \text { sen } \vartheta, \\
& \mathbf{H}_{1 \vartheta}=\mathbf{H}_{2 \vartheta}=\frac{2 \mu_{1}}{\mu_{1}+\mu_{2}} \mathbf{H}_{o} \cos \vartheta-\frac{2 \mathbf{J}}{c} \frac{1}{\mathrm{R}} .
\end{aligned}
$$

\section{Forza ponderomotrice esercitata sulla corrente per sezione unitaria.}

Per la determinazione della forza ponderomotrice esercitata su ogni sezione di cilindro di lunghezza 1 procediamo prima in questo modo: integriamo le forza agente sull' unità di volume sulla porzione di cilindro considerata ed aggiungiamo la risultante delle pressioni fittizie agenti sulla corrispondente superficie rispettivamente dalla parte di ciascun mezzo. 
a) Secondo Maxwell ed Einstein - Laub.

Dalla (3a), ponendo in essa $\mathbf{i}=\frac{1}{\pi \mathrm{R}^{2}} \mathbf{J}$, abbiamo pei punti interni al cilindro, tenendo conto che per le (9a) $\mathbf{H}_{2 x}$ e $\mathbf{H}_{2 z}$ sono indipendenti rispettivamente $\mathrm{da} x$ e $\boldsymbol{z}$ e che $\dot{\mathrm{e}}$ $\mathbf{H}_{2 y}=0$,

$$
\begin{aligned}
& \mathbf{F}_{x}=\frac{\mu_{2}-1}{4 \pi} \mathbf{H}_{2 z} \frac{\partial \mathbf{H}_{2 x}}{\partial z}+\frac{\mathbf{J}}{c \pi \mathrm{R}^{2}} \mathbf{H}_{2 z}, \\
& \mathbf{F}_{y}=0, \\
& \mathbf{F}_{z}=\frac{\mu_{2}-1}{4 \pi} \mathbf{H}_{2 x} \frac{\partial \mathbf{H}_{2 z}}{\partial x}-\frac{\mathbf{J}}{c \pi \mathrm{R}^{2}} \mathbf{H}_{2 x} ;
\end{aligned}
$$

ossia, sostituendo direttamente per le componenti di $\mathbf{H}_{\mathbf{2}} \mathrm{i}$ valori dati dalle (9a) e tenendo conto della posizione (8)

$$
\begin{aligned}
& \mathbf{F}_{x}=\frac{\mu_{2}+1}{\pi \mathbf{R}^{2}} \frac{J}{c}\left\{\frac{\mu_{1}}{\mu_{1}+\mu_{2}} \mathbf{H}_{0}-\frac{J}{c} \frac{\rho}{R^{2}} \cos \vartheta\right\} \\
& \mathbf{F}_{y}=0, \\
& \mathbf{F}_{z}=-\frac{\mu_{2}+1}{\pi \mathbf{R}^{2}} \frac{J^{2}}{c^{2}} \frac{\rho}{\mathrm{R}^{2}} \operatorname{sen} \vartheta .
\end{aligned}
$$

Integriamo ora queste componenti sul volume di una sezione unitaria; la prima di queste componenti ci dà

$$
\int_{0}^{\mathrm{R}} \rho d \rho \int_{0}^{2 \pi} d \vartheta \mathbf{F}_{x}=\frac{1}{c} \frac{\mu_{1}\left(\mu_{2}+1\right)}{\mu_{1}+\mu_{2}} \mathbf{H}_{0} \mathbf{J}
$$

mentre gli integrali dęlle altre due si annullano.

La forza di-volume risultante quindi è diretta secondo l'asse $x$ ed il suo valore si può mettere sotto la forma

$$
\mathbf{P}=\mathbf{P}_{x}=\frac{1}{c} \frac{\mu_{2}+1}{\mu_{1}+\mu_{2}} \mathbf{B}_{\mathbf{1 0}} \mathbf{J}
$$

essendo $\mathbf{B}_{10}=\mu_{1} \mathbf{H}_{0}$ l'induzione magnetica all'esterno del cilindro del campo primitivo. 
Vediamo ora quale contributo portano a questa risultante le tensioni superficiali.

Dalle (7) abbiamo che la somma delle forze agenti sull'unità di superficie $\dot{e}$, tenuto conto che sulla superficie vale la (11),

$$
\mathbf{T}_{1}+\mathbf{T}_{2}=\frac{1}{8 \pi}\left\{2\left(\mathbf{H}_{2}-\mathbf{H}_{1}\right)\left(\mathbf{B}_{2} \mathbf{n}\right)+\left(\mathbf{H}_{1}{ }^{2}-\mathbf{H}_{2}{ }^{2}\right) \mathbf{n}\right\} .
$$

Se teniamo conto poi che per la continuità in superficie è

$$
\mathbf{H}_{2}-\mathbf{H}_{1}=\mathbf{n}\left(\frac{1}{\mu_{2}}-\frac{1}{\mu_{1}}\right)\left(\mathbf{B}_{2} \mathbf{n}\right), \mathbf{H}_{1}{ }^{2}-\mathbf{H}_{2}{ }^{2}=\left(\frac{1}{\mu_{1}^{2}}-\frac{1}{\mu_{2}^{2}}\right)\left(\mathbf{B}_{2} \mathbf{n}\right)^{2},
$$

possiamo scrivere

$$
\mathbf{T}_{1}+\mathbf{T}_{2}=\frac{\mathbf{n}}{8 \pi}\left\{2\left(\frac{1}{\mu_{2}}-\frac{1}{\mu_{1}}\right)+\frac{1}{\mu_{1}^{2}}-\frac{1}{\mu_{2}^{2}}\right\}\left(\mathbf{B}_{2} \mathbf{n}\right)^{2} .
$$

Essendo, come abbiamo detto, $\mathbf{n}$ normale e diretto verso l'esterno alla superficie del cilindro è $\left(\mathbf{B}_{2} \mathbf{n}\right)=\mathbf{B}_{2 p}$ e per la (11)

$$
\mathbf{B}_{2 \rho}^{2}=4\left(\frac{\mu_{1} \mu_{2}}{\mu_{1}+\mu_{2}}\right)^{2} \mathbf{H}_{0}{ }^{2} \operatorname{sen}^{2} \vartheta
$$

Integrando allora le componenti di $\mathrm{T}_{1}+\mathrm{T}_{2}$ sulla superficie ognuna di esse da un contributo nullo perchè $\int_{0}^{2} \operatorname{sen}^{2} \vartheta \cos \vartheta d \vartheta, \int_{0}^{2 \pi} \operatorname{sen}^{3} \vartheta d \vartheta$ che compaiono nella risultante secondo l'asse $x$ e l'asse $z$ sono nulli, è perchè la risultante secondo l'asse $y$ è pure nulla essendo sempre $\mathbf{T}_{1}+\mathbf{T}_{2}$ perpendicolare a questo asse.

Concludendo allora possiamo asserire che partendo dalle formule di Maxwell ed Einstein-Laub, la forza ponderomotrice agente su ogni sezione unitaria del conduttore è diretta secondo l'asse $x$, normale quindi alla corrente e al campo magnetico primitivo, ed è espressa dalla (13). 
Nel caso particolare in cui il mezzo esterno ha permeabilità magnetica eguale a 1 , cioè nel caso in cui questo mezzo è l'etere la (13) diventa

$$
\mathbf{P}=\mathbf{P}_{x}=\frac{1}{c} \mathbf{H}_{0} \mathbf{J} .
$$

b) Secondo Hertz.

La forza agente sull' unita di volume è in questo caso data dalla (6a), che per $\mathrm{i}$ punti interni al conduttore, essendo ivi $\mu=\mu_{2}=$ cost., diventa

$$
\mathbf{F}^{\prime}=\frac{1}{c \pi \mathbf{R}^{8}}\left[\begin{array}{ll}
\mathbf{J} & \left.\mathbf{B}_{2}\right]
\end{array}\right.
$$

Per le (9 a) e la (8) le componenti di questa forza sono

$$
\begin{aligned}
& \mathbf{F}_{x}^{\prime}=\frac{1}{c} \frac{2 \mu_{1} \mu_{2}}{\mu_{1}+\mu_{2}} \frac{1}{\pi R^{2}} \mathbf{H}_{0} \mathbf{J}-\frac{\mu_{2}}{c^{2}} \frac{2 \mathbf{J}^{2}}{\pi \mathrm{R}^{4}} \rho \cos \vartheta \\
& \mathbf{F}_{y}^{\prime}=0 \\
& \mathbf{F}_{z}^{\prime}=-\frac{\mu_{2}}{c^{2}} \frac{2 \mathbf{J}^{2}}{\pi \mathrm{R}^{4}} \rho \operatorname{sen} \vartheta .
\end{aligned}
$$

Integrando queste componenti sul volume di una sezione unitaria si vede subito che solo il primo termine del secondo membro di $\mathbf{F}_{x}^{\prime}$ non si annulla, mentre i contributi secondo gli altri due assi vanno a zero: resta quindi sola una risultante secondo l'asse delle $x$

$$
\int_{0}^{\mathrm{R}} \rho d \rho-\int_{0}^{2 \pi} d \vartheta \mathrm{F}^{\prime}=\frac{1}{c} \frac{2 \mu_{1} \mu_{2}}{\mu_{1}+\mu_{2}} \mathbf{E}_{0} \mathbf{J} .
$$

Determiniamo la risultante delle pressioni fittizio in superficie.

La forza agente sull' unità di superficie da una parte e dall'altra dei due mezzi è per le (7a) e per le note continuità in superficie: 


$$
\mathbf{T}_{1}^{\prime}+\mathbf{T}_{2}^{\prime}=\frac{1}{8 \pi}\left\{\left(\mathbf{H}_{2}-\mathbf{H}_{1}\right)\left(\mathbf{B}_{2} \mathbf{n}\right)+\left[\left(\mathbf{B}_{2}-\mathbf{B}_{1}\right)\left[\mathbf{H}_{2} \mathbf{n}\right]\right]\right\}
$$

a questa si può dare anche la forma, essendo

$$
\begin{aligned}
& \mathbf{H}_{2}-\mathbf{H}_{1}=\mathbf{n}\left(\frac{1}{\mu_{2}}-\frac{1}{\mu_{1}}\right)\left(\mathbf{B}_{2} \mathbf{n}\right), \\
& \mathbf{B}_{2}-\mathbf{B}_{1}=\left(\mu_{2}-\mu_{1}\right)\left[\mathbf{H}_{2} \mathbf{n}\right] ; \\
& \mathbf{T}_{1}=\mathbf{T}_{2}^{\prime}=\frac{\mathbf{n}}{8 \pi}\left\{\left(\frac{1}{\mu_{2}}-\frac{1}{\mu_{1}}\right)\left(\mathbf{B}_{2} \mathbf{n}\right)^{2}+\left(\mu_{2}-\mu_{1}\right)\left[\mathbf{H}_{2} \mathbf{n}\right]^{2}\right\} .
\end{aligned}
$$

Integrando questa forza sulla superficie, per quanto abbiamo visto nel caso precedente di Maxwell, il termine che contiene $\left(\mathbf{B}_{2} \mathbf{n}\right)^{2}$ non dà alcun contributo. Resta quindi a considerare solo quel termine che contiene

$$
\begin{aligned}
{\left[\mathbf{H}_{2} \mathbf{n}\right]^{2}=\mathbf{H}^{2}=\frac{4 \mu_{1}{ }^{2}}{\left(\mu_{1}+\mu_{2}\right)^{2}} } & \mathbf{H}_{0}{ }^{2} \cos ^{2} \vartheta+ \\
& +\frac{4 \mathbf{J}^{2}}{c^{2} \mathbf{R}^{2}}-\frac{8 \mu_{1}}{\mu_{1}+\mu_{2}} \frac{\mathbf{H}_{0} \mathbf{J}}{c \mathrm{R}} \cos \vartheta .
\end{aligned}
$$

se teniamo conto del valore di $\mathbf{H}_{2\}}$ in superficie dato dalla (12).

Queste espressioni dipendono esclusivamente da $\vartheta$ e poichè $\mathbf{T}_{1}+\mathbf{T}_{2}^{\prime} \dot{e}$ un vettore normale alla superficie del conduttore le sue componenti secondo gli assi $x$ e $z$ si ottengono moltiplicando il suo valore rispettivamente per $\cos \vartheta \theta$ sen $\vartheta$.

Integrando allora sulla superficie di una sezione unitaria si ottiene

$$
\mathbf{R} \int_{0}^{2 \pi}\left(\mathbf{T}_{1 x}^{\prime}+\mathbf{T}_{2 x}^{\prime}\right) d \vartheta=\frac{1}{c} \frac{\mu_{1}\left(\mu_{1}-\mu_{2}\right)}{\mu_{1}+\mu_{2}} \mathbf{H}_{0} \mathbf{J}
$$

gli integrali delle altre due componenti sono nulli.

Quindi anche la risultante delle forze di superficie ̀̀ diretta secondo l'asse $x$. Sommando la (15) colla (16) otteniamo la forza totale agente su ogni sezione unitaria del conduttore

$$
\mathbf{P}^{\prime}=\mathbf{P}_{x}^{\prime}=\frac{1}{c} \frac{2 \mu_{1} \mu_{2}+\mu_{1}\left(\mu_{1}-\mu_{2}\right)}{\mu_{1}+\mu_{2}} \mathbf{H}_{0} \mathbf{J},
$$


ossia

$$
\mathbf{P}^{\prime}=\mathbf{P}_{x}^{\prime}=\frac{1}{c} \mathbf{B}_{10} \mathbf{J}
$$

Come ho gia accennato la risultante delle forze ponderomotrici si ottiene anche integrando sulla superficie del conduttore la pressione esercitata dal campo esterno. Come controprova dei risultati ottenuti ci basterà provarlo pel caso di Maxwell. In tal caso la detta pressione per unità di superficie ha per componente secondo l'asse $x(7)$

$$
\mathbf{T}_{1 x}=\frac{1}{8 \pi}\left\{2 \mathbf{H}_{1 x} \mathbf{B}_{1 p}-\mathbf{H}_{1}{ }^{2} \cos \vartheta\right\}
$$

e per le (9) e (11)

$$
\begin{aligned}
& \mathbf{T}_{\mathbf{1} x}=\frac{1}{4 \pi}\left\{\mathrm{K} \mathbf{H}_{0} \operatorname{sen} 2 \vartheta+\frac{2 \mathbf{J}}{c} \frac{\operatorname{sen} \vartheta}{\mathbf{R}}\right\} \frac{2 \mu_{1} \mu_{2}}{\mu_{1}+\mu_{2}} \mathbf{H}_{0} \operatorname{sen} \vartheta- \\
& -\frac{1}{8 \pi}\left\{(\mathbf{K}+1)^{2} \mathbf{H}_{0}{ }^{2} \operatorname{sen}^{2} \vartheta+\left[(\mathrm{K}-1) \mathbf{H}_{0} \cos \vartheta+\frac{2 \mathbf{J}}{c \mathbf{R}}\right]^{2}\right\} \cos \vartheta
\end{aligned}
$$

Integrando sulla superficie di una sezione unitaria solo $i$ termini contenenti $\operatorname{sen}^{2} \vartheta$ e $\cos ^{2} \vartheta$ porteranno un contributo diverso da zero; abbiamo quindi tenendo conto anche della (8).

$$
\mathrm{R} \int_{0}^{2 \pi} \mathbf{T}_{1 x} d \vartheta=\frac{1}{c} \frac{\mu_{1} \mu_{3}}{\mu_{1}+\mu_{2}} \mathbf{H}_{0} \mathbf{J}+\frac{1}{c} \frac{\mu_{1}}{\mu_{1}+\mu_{2}} \mathbf{H}_{0} \mathbf{J}
$$

$$
=-\frac{1}{c} \frac{\mu_{2}+1}{\mu_{1}+\mu_{2}} \mathbf{B}_{10} \mathbf{J} \text {. }
$$

Poichè $\mathbf{T}_{1 y}$ è nullo anche la sua risultante sulla superficie del cilindro è nulla, e poichè la espressione di $\mathbf{T}_{1 z}$ analoga a quella di $\mathbf{T}_{1 x}$ risulta composta da termini affetti da uno dei fattori $\cos 2 \vartheta \operatorname{sen} \vartheta, \cos \vartheta \operatorname{sen} \vartheta, \operatorname{sen}^{3} \vartheta, \cos ^{2} \vartheta \operatorname{sen} \vartheta$ anche il sno integrale sulla superficio scompare. La risultante della forza agente sulla sezione unitaria è diretta secondo l'asse $x$ è la sua espressiono (17) coincide con quella trovata per altra via (13). 


\section{Confronto dei risultati ottenuti.}

La forza ponderomotrice agente su ogni sezione unitaria del cilindro tanto secondo Maxwell che Hertz è diretta secondo l'asse $x$ e quindi normale al campo magnetico e alla corrente, e il valore di essa secondo Maxwell sta al valore secondo Hertz nel rapporto

$$
\frac{\mathbf{P}}{\mathbf{P}^{\prime}}=\frac{\mu_{2}+1}{\mu_{1}+\mu_{2}} .
$$

Le due espressioni quindi coincidono per $\mu_{1}=1$ come del resto si vede direttamente confrontando la (14) con la (13a). Hertz

Ora osserviamo che l' espressione della forza secondo

$$
\mathbf{P}^{\prime}=\frac{1}{c} \mathbf{B}_{10} \mathbf{J}
$$

è omogenea rispetto alla permeabilità, mentre non lo è quella di Maxwell

$$
\mathbf{P}=\frac{1}{c} \frac{\mu_{2}-1}{\mu_{1}+\mu_{2}} \mathbf{B}_{10} \mathbf{J}
$$

questa omogeneità di $\mathbf{P}^{\prime}$ sta in favore della sua maggiore attendibilità su $\mathbf{P}$.

Inoltre $\mathbf{P}^{\prime}$ non dipende punto dalla permeabilità magnetica del mezzo conduttore ma unicamente da quella del mezzo esterno per tramite di $\mathbf{B}_{10}$. Questo vien confortato anche dalle seguenti considerazioni. Se invece di un circuito rettilineo indefinito, come nel nostro problema, si prende a considerare un generico circuito chiuso, posto in un campo magnetico qualunque, percorso da una corrente continua, spostando il circuito nel campo il lavoro eseguito dalle forze del campo è proporzionale, oltre che all' intensità della corrente, al flusso magnetico che attraversa la superficie determinata dal circuito. Ma nel movimento del circuito possiamo ben supporre che il campo magnetico indotto dalla discontinuità di $\mu$ sulla superficie del conduttore e quello indotto 
dalla corrente si spostino assieme al circuito e quindi nel computo del flusso magnetico attraversante il circuito si debba tener conto solamente di quello che dipende dal campo primitivo esterno, di quello ciò̀ che secondo le nostre notazioni dipende da $\mathbf{B}_{10}$.

In ogni caso in generale la differenza che passa tra $\mathbf{P}$ e $\mathbf{P}^{\prime}$ è molto piccola perchè la permeabilità magnetica $\mu_{1}$ del mezzo esterno, trattandosi di un dielettrico, di poco differisce dall'unità e quindi anche il rapporto $\mathbf{P}$ : P' è molto prossimo all' unità.

Molto difficilmente quindi si potrà verificare sperimentalmente quale delle due espressioni $\mathbf{P} \circ \mathbf{P}^{\prime}$ sia più attendibile, tanto più che la misura delle forze ponderomotrici è difficile e difficilmente si possono eliminare le cause d'errore.

L'argomento di questa nota mi fu proposto dal prof. M. Abraham cui devo anche far vivi ringraziamenti pei validi suggerimenti datimi.

Milano, maggio 1911. 